\title{
Role-based Recommendation and Trust Evaluation
}

\author{
Yan Wang \\ Department of Computing \\ Macquarie University \\ Sydney, NSW 2109, Australia \\ yanwang@ics.mq.edu.au
}

\author{
Vijay Varadharajan \\ Department of Computing \\ Macquarie University \\ Sydney, NSW 2109, Australia \\ vijay@ics.mq.edu.au
}

\begin{abstract}
In general, in most trust evaluation systems, the evaluation of a target party under investigation relies on the trust ratings from responding peers/parties who have direct interactions with the target party. This involves recommendations and the trust evaluation on recommendations. The evaluation on the recommendations by the recommendation receiver depends on a few factors, which include the roles of the recommender in the domain of the target being recommended, the recognition of these roles by the recommendation receiver, and the recommendation reputation (trust) of the recommender. In this paper, we propose a novel role-based recommendation and trust evaluation framework (RBRTE), which takes the above factors into account.
\end{abstract}

Keywords: recommendation, trust, role, role hierarchy and domain.

\section{Introduction}

Trust and recommendation evaluation is an important issue in many applications, such as Peer-to-Peer systems [5, 21], e-commerce systems [7], e-service systems [16], education system, and job seeking systems etc.

For instance, Peer-to-Peer (P2P) network is a typical application where trust evaluation plays an important role. In P2P networks, each peer can play the role of a client and a service provider at the same time. In a typical P2P architecture, peers communicate with each other directly without intermediate servers. Currently this type of popular infrastructure is being applied to information sharing networks, such as GNutella [2].

In such an environment, before interacting with an unknown peer, it is rational to doubt its trustworthiness. Therefore, it makes the new transaction securer to enable the trust evaluation prior to the interactions with an unknown peer.
In Peer-to-Peer (P2P) environments, the trust evaluation on an unknown peer relies on the ratings and recommendations by other peers, which have transaction history with the target peer that is being investigated. If the unknown target peer is one of potential sellers, the end-peer (requesting peer) can enquire other peers about the target peer's transaction trust. After having collected the feedback from a set of responding peers, the requesting peer can analyze the data and evaluate the trust status of the target peer [8, 12, 21].

Actually, in some sense, the social society is a Peer-toPeer network, where some people interact with each other directly. In the social P2P society, people can recommend an object to other people. The object may be a movie, a job, a product, a book, or a person etc.

In addition, reputation-based trust evaluation is also an important issue in B2B and B2C e-commerce systems [7] and service-oriented environments [16]. In all these cases, the trust evaluation relies heavily on recommendations. Moreover, recommendation is a kind of e-services (e.g. in recommendation systems) [20]. If the recommendation receiver receives recommendations from different recommenders, impacts should be evaluated. On the other hand, if a recommender recommends different types of objects to the same person, these recommendations from the same recommender may have different impacts on the decision made by the recommendation receiver. Therefore, how to evaluate a recommendation remains an issue to be explored.

In this paper, we present a novel role-based framework for evaluating recommendations, where the role of the recommender in the domain of the target being recommended is a key factor for determining the trust on recommendations. The trust also results from the recognition of these roles by the recommendation receiver. Meanwhile, we will also discuss how to determine the recommendation reputation (credibility) of a recommender.

This paper is organized as follows. In section 2, we review some existing studies. Section 3 presents the rolebased framework for recommendation evaluation while the role set and the role hierarchy are introduced in Section 
4. Section 5 discusses the method of computing the trust on recommendations. Section 6 presents some applications where the proposed framework can be applied. Finally Section 7 concludes our work.

\section{Related Work}

The computation of trust drew some attention over 10 years ago. The work by S. March [11] is widely considered as the earliest study on trust formalization. The work by Berth et al [4] is also one of the earliest studies. This work presents the notions of direct trust, recommendation trust and derived trust, which is derived from the direct trust and recommendation trust.

Trust evaluation is also an important topic in Peer-toPeer (P2P) networks, which are based the infrastructure extending the traditional client/server architecture. In P2P networks, each peer can be a client and a service provider at the same time. P2P networks are widely utilized for information sharing systems, where each peer can download and upload files, such as GNutella [2] networks.

As the information sharing process may involve payment, the P2P network can be further extended to P2P e-commerce systems [3], which is actually the sort of Customer-to-Customer (C2C) e-commerce applications without central servers.

eBay [1] is a typical Customer-to-Customer (C2C) ecommerce system with central management, where peers - buyers or sellers - can evaluate each other based on the service quality or behaviors during the transactions. These evaluations are posted on the central server and thus are released to the public. Each evaluation can be taken as the recommendation of the peer to be evaluated though the result may be quite negative.

Due to the special infrastructure of $\mathrm{P} 2 \mathrm{P}$ networks, trust evaluation remains a challengeable issue which draws much attention in the research community.

In [5], Damiani et al proposed XRep: a reputation-based approach for evaluating the reputation of peers through distributed polling algorithm before downloading any information. EigenTrust [8] collects the local trust values of all peers to calculate the global trust value of a given peer. In [12], Marti et al proposed a voting reputation system that collects responses from other peers on a given peer. The final reputation value is calculated combining the values returned by responding peers and the requesting peer's experience with the given peer. This seems more reasonable than the model in [5]. However, this work and the work in [8] don't explicitly differentiate transaction reputation and recommendation reputation.

In [17] Wang et al proposed several trust metrics for the trust evaluation in a decentralized environments (e.g. P2P) where a trust value is a probabilistic value in the scope of
$[0,1]$. Prior to the interaction with an unknown peer $P_{x}$, the end-peer collects other peers' trust evaluations over $P_{x}$. A method has been proposed for trust modification after a series of interactions with $P_{x}$ that a good value results from the cumulation of constant good behaviors leading to a series of constant good trust values. In [18] Wang et al proposed a method to measure the recommendation trust (referred to as credibility in [18]), which is based on the requesting peer's interaction experience with the target peer and the recommendations of responding peers in multiple rounds.

In the literature, trust issue also caused much attention which is not bound to the P2P networks only. In [14], Sabater and Sierra proposed a model discussing the trust development between groups. In [6], Griffiths proposed a multi-dimensional trust model which allows the agents to model the trustworthiness of others according to various criteria. In [13], Ray et al proposed a generic vector trust model for developing trustworthy systems. In [10], Lin et al proposed and a method of reputation-based trust evaluation in service-oriented environments based on the proposed architecture consisting of distributed trust management brokers. In [16], Vu et al proposed a model to evaluate and rank the reputation of QoS-based services.

Nevertheless, in most existing studies, the final trust evaluation relies on the ratings of peers or parties who have interaction history with the target peer being investigated and send these ratings to the requesting peer or party. Therefore these responding peers or parties may have different impact on the final trust evaluation due to their roles. Namely, when evaluating the trust of the target peer based on recommendations, the factors of each recommender should be taken into account explicitly.

\section{Recommendation and Role-based Trust Evaluation}

In this section, some basic notions on trust will be introduced. Then we will present the generic role-based trust evaluation framework staring from some scenarios in real life.

\subsection{Trust}

Similar to the definition in [9], we define the reputationbased trust between two parties to be the extent to which a party believes that the other party is willing and able to act (e.g. provide some services) or posses some properties in the other party's interests.

The trust relationship can be extended to describe the extent to which a party prefers another in a certain situation. This relationship depends on the preference of the recommending party and the merits of the recommended party. 
For instance, a young man Peter prefers a MAZDA MX-5 sports car due to its merits and sports performance.

Trust can be measured and represented by a value as the result of an evaluation. In this work, we let $T_{A \rightarrow B}$ denote the trust value given by party $A$ to party $B$, which is real number in a scope of $[0,1]$, where the higher the value is, the better the trust is. The value of $T_{A \rightarrow B}$ can be obtained after the direct interaction between $A$ and $B$, under which circumstance the trust is called a direct trust. In our work, $B$ can be an active object (e.g. a person) or an inactive object (e.g. a book). In the above example, Peter gives 1.0 to his favorite car and recommends his opinion to his friends.

\subsection{Recommendation}

Generally a recommendation occurs between a recommender $r_{c}$ and a recommendation receiver $r_{v}$ who typically may not know beforehand the target $x$ being recommended. Namely, it is the recommender $r_{c}$ to evaluate the target $x$. The evaluation $T_{r_{c} \rightarrow x}$ can be represented as a value in $[0,1]$. This value is the measurement of the direct trust of $r_{c}$ over $x$. It is a local value to $r_{c}$. But it can be sent to $r_{v}$ as the request of $r_{v}$ or it can be broadcasted by $r_{c}$ to the network. Thus, from the point view of the value receiver, it is the recommended trust value over $x$.

A simple recommendation framework can be described in Definition 1.

Definition $1\left(R M_{1}\right)$ : A recommendation framework can be represented as a 4-tuple:

$$
R M_{1}=<r_{c}, r_{v}, x, T_{r_{c} \rightarrow x}>
$$

Target $x$ can be an object in general, such as a book, a movie or a person.

\subsection{Trust on Recommendation}

Typically $T_{r_{c} \rightarrow x}$ reflects the extent to which $r_{c}$ prefers target $x$ and it is local to $r_{c}$. Once the recommendation receiver $r_{v}$ receives $T_{r_{c} \rightarrow x}$ as a recommendation from the recommender, she/he may not take the recommendation value as the trust value on $x$ directly as this depends on the extent to which $r_{v}$ trusts $r_{c}$ 's recommendation.

In general, here we can assume that $r_{v}$ doesn't know target $x$ and $r_{v}$ receives the recommendation from $r_{c}$ only. We can also assume the trust value given by $r_{v}$ over $x$ is:

$$
T_{r_{v} \rightarrow x}=g\left(T_{r_{v} \rightarrow r_{c}}, T_{r_{c} \rightarrow x}\right)
$$

where $T_{r_{v} \rightarrow r_{c}} \in[0,1]$ is the trust given by $r_{v}$ over $r_{c}$ 's recommendation $T_{r_{c} \rightarrow x}$. $T_{r_{v} \rightarrow r_{c}}$ is the measure of the extent to which $r_{v}$ trusts $r_{c}$ 's recommendation. Namely, it is the recommendation trust.

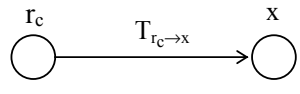

$\mathrm{T}_{\mathrm{r}_{\mathrm{c}} \rightarrow \mathrm{x}}$ is a local trust/rating

(a)

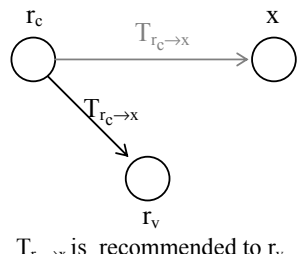

(b)

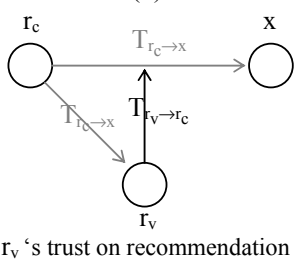

(c)

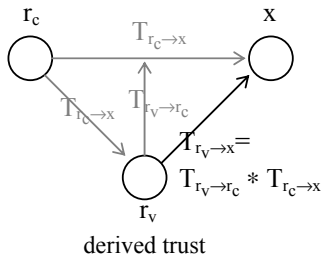

(d)

Figure 1. Triple Trust Relations

In the simplest case, we can assume that

$$
T_{r_{v} \rightarrow x}=g\left(T_{r_{v} \rightarrow r_{c}}, T_{r_{c} \rightarrow x}\right)=T_{r_{v} \rightarrow r_{c}} * T_{r_{c} \rightarrow x}
$$

The relationship of recommendation trust and recommended trust is depicted in Figure 1. $T_{r_{v} \rightarrow x}$ is the derived trust as described in [4].

Therefore an improved recommendation framework can be described as follows.

Definition $2\left(R M_{2}\right)$ : A recommendation framework can be represented as a 6-tuple:

$$
R M_{2}=<r_{c}, r_{v}, x, T_{r_{c} \rightarrow x}, T_{r_{v} \rightarrow r_{c}}, T_{r_{v} \rightarrow x}>
$$

\subsection{Role-based Evaluation on Recommendations}

In $R M_{2}$, how to calculate $T_{r_{v} \rightarrow r_{c}}$ remains an issue. In our framework, the role of the recommender is an important factor to be taken into account. Now let's first consider the following scenarios.

Scenario 1:

Professor Alex is a well-known expert on computer security. He is teaching a subject on "Network Security" to 
undergraduates. He recommended a textbook $B$ on network security to his students.

In this scenario, in general, each student trusts Alex's recommendation very well because of Alex's role (subject lecturer), his expertise (e.g. professor position) and the domain of the target to be recommended.

\section{Scenario 2:}

Professor Alex is an expert on computer security. He is teaching a subject on "Network Security" to undergraduates. He likes music very much. But he is not an expert. He recommended a pop music $\mathrm{CD}$ to his students.

In this scenario, Alex's recommendation is not as trustworthy as the one in scenario 1 because music is not his expertise. His roles as a subject lecturer and a computer science professor are not as important as scenario 1 .

\section{Scenario 3:}

Professor Alex is teaching a subject on "Network Security" to undergraduates. In his class, student $S$ recommends a textbook on computer security to student $V$.

In this scenario, in general, $S$ ' recommendation is not as trustworthy as Alex's recommendation in scenario 1.

In the above scenarios, student $V$ has different trust values (levels) on the recommendations that were given by different persons for different targets. The difference comes from the role(s) of the recommender, the domain of the recommended target, and the recommender's expertise level in the domain.

Therefore, a more generic recommendation framework can be described as follows.

Definition 3: A recommendation framework can be represented as an 8-tuple:

$R M_{3}=<r_{c}, r_{v}, x, T_{r_{c} \rightarrow x}, \vec{r}_{r_{c}}, D_{x}, T_{r_{v} \rightarrow r_{c}}, T_{r_{v} \rightarrow x}>$

where

- $r_{c} \in R_{C}$ is the recommender, $R_{C}$ is the set of recommenders;

- $r_{v}$ is the recommendation receiver;

- $x$ is the target being recommended;

- $T_{r_{c} \rightarrow x} \in[0,1]$ is the recommendation given by $r_{c}$ over target $x$;

- $\vec{r}_{r_{c}} \in R_{O}$ is the role of the recommender, $R_{O}$ is the set of roles of recommenders;

- $D$ is the domain of targets to be recommended. The domain of $x$ is denoted as $D_{x} \in D$;

- $R_{C} 2 R_{O} \subseteq R_{C} \times R_{O}$ is a many-to-many recommender to role assignment relation. Each $r_{c}$ may have different roles. On the other hand, each role may correspond to different recommenders;
- $R_{O} 2 D \subseteq R_{O} \times D$ is a many-to-many role to domain relation. There are several roles in a certain domain. Meanwhile, each role may correspond to different domains;

- $h:\left(R_{C}, D\right) \rightarrow R_{O}$ is a function mapping $R_{C}$ and $D$ to a role. That is, given $r_{c} \in R_{C}$ and $D_{x} \in D, \vec{r}_{r_{c}}=$ $h\left(r_{c}, D_{x}\right) \in R_{O}$, where $\left(r_{c}, h\left(r_{c}, D_{x}\right)\right) \in R_{C} 2 R_{O}$ and $\left(D_{x}, h\left(r_{c}, D_{x}\right)\right) \in R_{O} 2 D$.

According to the above-mentioned scenarios, a recommender may have various expertise levels in different domains. These expertise levels lead to different roles in the domain. A role with a higher expertise level in a domain should be trusted more than a role with a lower expertise level in the same domain. Namely, different roles of a recommender have different impacts on recommendations.

\section{Role Set and Role Hierarchy}

As discussed in Definition 3, role is a core notion in our recommendation framework. Here role is somewhat relevant to but different from the notion in RBAC (RoleBased Access Control) [15] and social network analysis [19], where a role refers to the patterns of relations which obtain between social positions. To be more concisely, we provide the definition as follows.

Definition 4: In the recommendation evaluation framework, the role of a recommender is defined to be the social position, title or rank reflecting the expertise level of the recommender and the impact level of the recommender's recommendations in the domain of the target to be recommended.

\subsection{Total Order Role Set}

In an ideal case, we can assume a total order role set where there are different roles reflecting different expertise levels. Particularly, in terms of recommendation impact, these roles are fully ordered. For instance, suppose Prof. Alex and Dr. Brown with a Lecturer position are sharing the teaching of subject "Network Security". Their roles are in the total order role set $\{$ Professor, Associate Professor, Senior Lecturer, Lecturer, Associate Lecturer $\}$, where Professor $>$ Associate Professor $>$ Senior Lecturer $>$ Lecturer $>$ Associate Lecturer with respect to the expertise levels in the domain. If they fully recommend two different textbooks (i.e. $T_{\text {Alex } \rightarrow \text { book } 1}=T_{\text {Brown } \rightarrow \text { book } 2}=1$ ) to students respectively, Prof. Alex's recommendation will have more impact on students as the role of a Professor has more impact than the role of a Lecturer.

In general, we describe the relationship between them in the following definition. 
Definition 5: Let $R_{O}$ and $D_{x}$ denote the fully ordered role set and the domain of target $x$. Let $\vec{r}_{r_{c}}\left(D_{x}\right)$ denote the role of $r_{c}$ in domain $D_{x} . \theta:\left(R_{O}, D\right) \rightarrow I$ is the impact function mapping $R_{O}$ and $D$ to $I=\{\mu: \mu$ is the impact factor and a real number $\in[0,1]\}$, which reflects the expertise level and thus is the impact set of the recommendation resulting from $r_{c}$ 's role in domain $D_{x}$.

With the definition of impact function $\theta$, we can compare two roles in the same role set.

Definition 6: Given two roles $\overrightarrow{r_{1}}$ and $\overrightarrow{r_{2}}$ in a role set $R_{O}$ for domain $D_{x}, \overrightarrow{r_{1}}<\overrightarrow{r_{2}}$ iff $\theta\left(\overrightarrow{r_{1}}, D_{x}\right)<\theta\left(\overrightarrow{r_{2}}, D_{x}\right)$.

Definition 7: Given two roles $\overrightarrow{r_{1}}$ and $\overrightarrow{r_{2}}$ in a role set $R_{O}$ for domain $D_{x}, \overrightarrow{r_{1}}=\overrightarrow{r_{2}}$ iff $\theta\left(\overrightarrow{r_{1}}, D_{x}\right)=\theta\left(\overrightarrow{r_{2}}, D_{x}\right)$.

According to Definition 5 and Definition 6, given two roles $\overrightarrow{r_{1}}$ and $\overrightarrow{r_{2}}$ in a role set $R_{O}$ for domain $D_{x}$, $\overrightarrow{r_{1}} \leq \overrightarrow{r_{2}}$ iff $\theta\left(\overrightarrow{r_{1}}, D_{x}\right) \leq \theta\left(\overrightarrow{r_{2}}, D_{x}\right)$.

\subsection{Role Hierarchy}

In all the above definitions, we simply assume that given a domain $D_{x}, r_{c}$ has a single role $\vec{r}_{r_{c}}$ based on $r_{c}$ 's position in $D_{x}$. However, in more general cases, $r_{c}$ may have different roles as $r_{c}$ has different positions in $D_{x}$. One or several positions may correspond to one role. All positions may correspond to several roles in the domain.

For instance, in scenario 1 introduced in Section 3.4, Prof. Alex has several roles:

1. a professor in Computer Science

2. a well-known expert on computer security, and

\section{3. "Network Security" lecturer}

A more generic definition of impact function $\theta$ is defined as follows.

Definition 8: Let $R_{O_{r_{c}\left(D_{x}\right)}}$ denote the role set of recommender $r_{c}$ in domain $D_{x}$. Namely $R_{O_{r_{c}\left(D_{x}\right)}}=$ $\left\{\vec{r}_{r_{c}\left(D_{x}\right)}^{(i)}: \vec{r}_{r_{c}\left(D_{x}\right)}^{(i)}\right.$ is one of $r_{c}$ 's roles in $\left.D_{x}\right\}$. Thus the impact function $\theta$ has the format as follows:

$$
\theta:\left(2^{R}, D\right) \rightarrow I
$$

where $I=\{\mu: \mu$ is a real number in $[0,1]\}$.

In this work, we assume a role hierarchy exists in a certain domain.

A role hierarchy example is illustrated in Figure 2 (a). In this figure, there are several layers. i.e., layers $A, B, C$ and $D$. Prof. Alex's roles are $B 1-C 1-D 1$ (refer to Figure 2 (b)). Other roles can be laid on top of layer $B$, such as Turing Award recipient or Nobel Prize recipient.

For the sake of simplicity, we assume the domain-based role hierarchy is a partially ordered set where each element reflects a level of expertise in the domain and the partial order relation $\leq$ exists between some elements. But

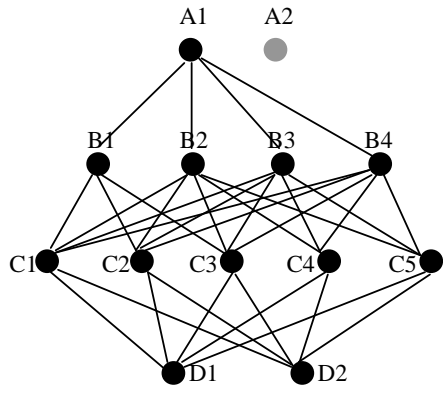

$\begin{array}{ll}\begin{array}{l}\text { A1: Turing Award } \\ \text { recipient }\end{array} & \begin{array}{l}\text { A2: Nobel Prize } \\ \text { recipient }\end{array} \\ \begin{array}{l}\text { B1: well-known } \\ \text { expert on security }\end{array} & \begin{array}{l}\text { B2: expert on } \\ \text { security }\end{array} \\ \begin{array}{l}\text { B3: CS expert } \\ \text { with knowledge }\end{array} & \begin{array}{l}\text { B4: CS expert } \\ \text { without knowledge }\end{array} \\ \text { security } & \text { security } \\ \text { C1: CS Professor } & \text { C2: CS Assoc. } \\ & \text { Professor } \\ \begin{array}{l}\text { C3: CS Senior } \\ \text { Lecturer }\end{array} & \text { C4: CS Lecturer } \\ \begin{array}{l}\text { C5: CS Assoc. } \\ \text { Lecturer }\end{array} & \\ \begin{array}{l}\text { D1: "Network } \\ \text { Security" Lecturer }\end{array} & \text { D2: "E-Commerce } \\ \text { Systems" Lecturer }\end{array}$

(a)

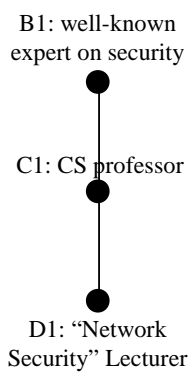

(b)

Figure 2. A Role Hierarchy Example

in a certain layer, the total order relation may exist, i.e., all elements in a layer form the fully ordered set. For instance, in layer $B$ : $B_{4} \leq B_{3} \leq B_{2} \leq B_{1}$, and layer $C$ : $C_{5} \leq C_{4} \leq C_{3} \leq C_{2} \leq C_{1}$. In a role hierarchy $R H$, if $\overrightarrow{r_{1}}, \overrightarrow{r_{2}} \in R H$, and $\overrightarrow{r_{1}} \leq \overrightarrow{r_{2}}$, that implies role $\overrightarrow{r_{1}}$ is not as important as role $\overrightarrow{r_{2}}$ with respect to the impact of recommendations in the domain.

However, the role hierarchy is dependant on the domain $D_{x}$. This feature can be reflected in several aspects.

1. Let $R$ denote the set of all roles. A role $\vec{r} \in R$ may not appear in a $R H_{D_{x}}$ of domain $D_{x}$.

For instance, Nobel Prize recipient is a role. But typically it is not likely to appear in a role hierarchy like the one in Figure 2 (a). However, if it is to recom- 
mend a person for applying a new academic position, that will be different. If the recommender is a Nobel Prize recipient, the positive recommendation will have strong impact.

2. If $\vec{r}_{r_{c}} \in R H_{D_{x}}, \vec{r}_{r_{c}}$ may not appear in $R H_{D_{y}}$.

For instance, Prof. Alex is also a classic music fan. Therefore he recommended a classic music CD to his students (scenario 2 in section 3.4), i.e. classic music fan is a role in $R H_{\text {music } C D}$.

However, this role has nothing to do with the case of recommending a textbook on computer security to students (scenario 1 in section 3.4). Therefore the role a classic music fan doesn't appear in the role hierarchy $R H_{\text {textbook }}$

3. Given two role hierarchies $R H_{1}$ and $R H_{2}$ with the same role set (i.e., $\forall \vec{r} \in R H_{1}$, then $\vec{r} \in R H_{2}$ and $\forall \vec{r} \in R H_{2}$, then $\vec{r} \in R H_{1}$ ) the partial order existing in $\mathrm{RH}_{1}$ may not exist in $\mathrm{RH}_{2}$.

For instance, Dr. Brown is a Lecturer teaching ecommerce. He also has 3 years' research experience on e-commerce systems. If the task is to recommend a textbook on e-commerce, Dr Brown's role (a CS Lecturer) is more important than Prof. Alex (a CS Professor) as Prof. Alex is not good at e-commerce field.

4. The above case also illustrates another feature. The role is an upper layer (e.g. $C 4$ ) may be not as important as a role in a lower layer (e.g. D2) in a certain domain.

\subsection{Role Comparison}

As a role hierarchy may be not a total order set, the roles of two recommenders are comparable under some conditions.

Definition 9: Assume there are two recommenders $r_{c_{1}}$ and $r_{c_{2}}$. We can compare their roles in domain $D_{x}$ in the following cases.

1. if both $r_{c_{1}}$ and $r_{c_{2}}$ have a single role in $R H_{D_{x}}$, and $\vec{r}_{c_{1}} \leq \vec{r}_{c_{2}}$, then $\theta\left(\vec{r}_{c_{1}}, D_{x}\right) \leq \theta\left(\vec{r}_{c_{2}}, D_{x}\right)$;

2. if $\forall \vec{r}^{(i)} \in R_{O_{r_{c_{1}}\left(D_{x}\right)}}, \exists \vec{r}^{(j)} \in R_{O_{r_{c_{2}}\left(D_{x}\right)}}$, and $\vec{r}^{(j)} \leq$ $\vec{r}^{(j)}$, then $R_{O_{r_{c_{1}}\left(D_{x}\right)}} \leq R_{O_{r_{c_{2}}\left(D_{x}\right)}}$.

Scenario 2 in Section 3.4 belongs to the second case. Both Prof. Alex and Dr. Brown have the role as the lecturer of subject "Network Security". As Professor > Lecturer, $R_{O_{\text {Alex }}\left(D_{x}\right)}>R_{O_{\text {Brown }}\left(D_{x}\right)}$.

\section{How to Compute Trust on Recommenda- tions}

Let $T_{r_{v} \rightarrow r_{c}}$ denote the trust of $r_{v}$ on $r_{c}$ 's recommendation to target $x$ (i.e. $T_{r_{v} \rightarrow x}$ ). $T_{r_{v} \rightarrow r_{c}}$ results from several factors.

1. $r_{v}$ 's knowledge in domain $D_{x}$;

2. $r_{v}$ 's recognition of $r_{c}$ 's role(s) in domain $D_{x}$;

3. $r_{c}$ 's recommendation credibility.

$r_{v}$ 's knowledge level in domain $D_{x}$ is important and helpful for $r_{v}$ to make accurate judgement on $T_{r_{c} \rightarrow x}$. Meanwhile, $r_{v}$ 's recognition on $r_{c}$ 's role(s) is critical for evaluating $T_{r_{c} \rightarrow x}$ objectively. There are two ways for the abovementioned two factors to impact role-based recommendation evaluation.

1. If there is a standard impact function $\theta, r_{v}$ 's knowledge in domain $D_{x}$ (denoted as $K_{r_{v}}\left(D_{x}\right)$ ) and $r_{v}$ 's recognition of $r_{c}$ 's roles in domain $D_{x}$ (denoted as $R g_{r_{v} \rightarrow \vec{r}_{r_{c}}}\left(D_{x}\right)$ ) can result in the recognition factor, i.e.,

$$
\lambda=f\left(K_{r_{v}}\left(D_{x}\right), R g_{r_{v} \rightarrow \vec{r}_{r_{c}}}\left(D_{x}\right)\right) \in[0,1]
$$

Thus, the final value will be $T_{r_{c} \rightarrow r_{v}}=\theta \cdot \lambda \in[0,1]$.

2. If the impact function $\theta$ is determined by $r_{v}$ it can take $K_{r_{v}}\left(D_{x}\right)$ and $R g_{r_{v} \rightarrow \vec{r}_{r_{c}}}\left(D_{x}\right)$ into account directly, i.e., $\theta:\left(R_{O}, D, K_{r_{v}}\left(D_{x}\right), R g_{r_{v} \rightarrow \vec{r}_{r_{c}}}\left(D_{x}\right)\right) \rightarrow I$. In such a case, $T_{r_{c} \rightarrow r_{v}}=\theta$ (assume $\lambda=1$ for Definition 10 below).

$r_{c}$ 's recommendation credibility (denoted as $C_{r_{r_{c}}}$ ) refers to the credibility or accuracy of $r_{c}$ 's recommendations. It is a direct factor to determine $r_{v}$ 's trust on $T_{r_{c} \rightarrow x}$ and thus impacts $T_{r_{v} \rightarrow x}$ (refer to equation (1)). If $r_{v}$ 's is familiar with $r_{c}, r_{v}$ can know $r_{c}$ 's recommendation credibility. Otherwise, it may take a few rounds of interaction for $r_{v}$ to know the recommendation credibility of $r_{c}$ [18] by comparing $r_{c}$ 's recommendations with other recommenders' recommendations and $r_{v}$ 's rating of the target $x$. Namely, $C_{r_{r_{c}}}$ is determined by

1. $r_{c}$ 's recommendations to target $x$ in multiple rounds;

2. $r_{v}$ 's experience and ratings with target $x$;

3. the deviations of $r_{c}$ 's recommendations in different rounds, which results from $r_{c}$ 's recommendations and $r_{v}$ 's rating to $x$. 
In principal, if $r_{c}$ 's recommendation is different from $r_{v}$ 's rating in multiple rounds, it will result in the bad recommendation credibility of $r_{c}$. In contrast, if $r_{c}$ 's recommendation is close to $r_{v}$ 's rating in multiple rounds, it will result in good recommendation credibility of $r_{c}$.

Definition 10: Let $T_{r_{v} \rightarrow r_{c}}$ denote the trust of $r_{v}$ on $r_{c}$ 's recommendation.

$$
T_{r_{v} \rightarrow r_{c}}=\mu \cdot \lambda \cdot C_{r_{c}}
$$

where

1. $\mu \in[0,1]$ is the impact factor resulting from $r_{c}$ 's role in the domain $D_{x}$;

2. $\lambda \in[0,1]$ is the recognition factor resulting from $r_{v}$ 's knowledge in domain $D_{x}$ and $r_{v}$ 's recognition of $r_{c}$ 's roles in domain $D_{x}$;

3. $C_{r_{c}} \in[0,1]$ is the recommendation credibility of $r_{c}$.

\section{Applications}

The proposed role based recommendation framework can be widely applied to many e-service systems. Here we list several examples.

\subsection{Role Certificate and Agent-based Evaluation System}

Here we assume an autonomous agent-based system incorporating the proposed framework. In the system each recommender can send its recommendation via his/her software agent. Meanwhile, the role information (e.g. role certificate) of each recommender can be obtained from the recommender, which can be verified with independent certificate issuing authorities. lows.

A role certificate can have a structure depicted as fol-

\section{Subject: Owner's name or ID;}

2. Role: The role of the certificate owner;

3. Issuer: The name of the authority issuing the certificate;

4. Serial Number;

5. Expiration Date;

6. Issuer's Digital Signature.

Based on the proposed framework, the software agent of the recommendation receiver can evaluate recommendations and compute trust values to the recommended target.

\subsection{Online Teaching Evaluation}

In most online teaching evaluation systems, where students rate their teachers in several aspects, basically all students are taken equally. However, if students can be divided into 3 categories, i.e., "good students", "average students" and "weak students", a typical phenomenon is that good students are likely to give good evaluations while weak students are likely to give low evaluations as they may have more difficult in learning. If the lecturer teaches much more details offering more help to weak students, the lecture may become tedious from the point view of other students.

Therefore, a solution is that the lecturer should be concentrating on "average" students. Meanwhile the system can assign different roles to students with different impacts for the teaching evaluation according to their GPAs when registering the subject where average students are given the highest impact factor.

\subsection{Job Seeking Recommendation}

In the job application process, typically recommendations from previous or current employers are essential. According to our framework, in order to have more impact, the recommender should have a higher position and close working relationship with the applicant (e.g. direct leader). In other words, the role of the recommender is important.

For instance, $X, Y$ and $Z$ are all applying a Lecturer position. Suppose each applicant has got a "very good" recommendation. But $X$ 's recommender is not famous in the field. $Y$ 's recommender is well-known while $Z$ 's recommender is famous and a Turing Award recipient. Therefore, it is the most beneficial to $Z$.

\subsection{E-commerce Applications}

\subsubsection{Example 1}

Suppose party $A$ and party $B$ are two independent recommendation systems existing for a few years [20]. $A$ was ranked 5-star by a third party with respect to commercial reputation. $B$ was rated as a 4-star system. Thus they have different impacts on customers in terms of the role and expertise levels. Namely, $\overrightarrow{r_{A}}>\overrightarrow{r_{B}}$ as $\theta\left(\overrightarrow{r_{A}}, D_{p}\right)>$ $\theta\left(\overrightarrow{r_{B}}, D_{p}\right)$ (refer to Definition 6) where $D_{p}$ is the domain of the product $p$ to be recommended.

\subsubsection{Example 2}

In P2P e-commerce environments, each buyer can rate the seller after a transaction based on the quality of the seller's service provided. If the seller $S$ is unknown to a buyer $B$, the buyer can enquire other buyers about the service quality 
and transaction trust of the seller. Here all existing buyers with transaction history with $S$ may have different roles with respect to their transaction experience with $S$. Namely, if a buyer $B_{i}$ has a lot of transactions with $S, B_{i}$ 's rating, which becomes a recommendation when sent to $B$, has more impact on $B$. On the contrary, if $B_{i}$ has just one transaction with $S$, its recommendation has the minimal impact.

\section{Conclusions}

In this paper, we proposed a framework of role-based recommendation and trust evaluation, which correlates social network, peer-to-peer networks and trust evaluation. In our framework, each role reflects a level of the recommender's expertise in the domain of the target being recommended. The impact results from the aggregation of all roles of the recommender in the domain of the target. In addition, the trust on the recommendation also depends on the recognition of the recommender by the recommendation receiver, which is related to the receiver's knowledge in the domain. Moreover, the recommendation trust of the recommender should be taken into account when calculating the derived trust. To our best knowledge, it is the first model to take the role of a recommender into account in reputationbased trust and recommendation evaluation.

For future work, several issues remain open. First, given domain $D_{x}$, it is important to organize a rational role hierarchy $R H_{D_{x}}$. As the recommender may has different roles in different layers of $R H_{D_{x}}$, a good role hierarchy will lead to the reasonable computation of the aggregation of roles and thus lead to a reasonable impact factor. Secondly, given a rational $R H$, how to aggregate roles and define a reasonable impact function remains an issue.

\section{References}

[1] eBay. http://www.eBay.com/.

[2] GNutella. http://www.gnutella.com/.

[3] S. Anancha, P. D'souza, F. Perich, A. Joshi, and Y. Yesha. $\mathrm{P} 2 \mathrm{P}$ M-commerce in pervasive environments. ACM SIGecom Exchange, 3(4):1-9, January 2003.

[4] T. Berth, M. Borcherding, and B. Klein. Valuation of trust in open networks. In Proceedings of the European Symposium on Computer Security (ESORICS), pages LNCS 875, 3-18, Brighton, UK, 1994. Springer-Verlag.

[5] E. Damiani, S. D. C. di Vimercati, S. Paraboschi, P. Samarati, and F. Violante. A reputation based approach for choosing reliable resources in peertopeer networks. In Proceedings of ACM CCS'02, pages 207-216, Washington DC, USA, November 2002.

[6] N. Griffiths. Task delegation using experience-based multidimensional trust. In Proceedings of the 4th International Joint Conference on Autonomous Agents in Multi-Agent Systems (AAMAS-05), pages 489-496, 2005.
[7] C. W. Holsapple and S. Sasidharan. The dynamics of trust in B2C e-commerce: a research model and agenda. Information Systems and E-Business Management, 3(4):377-403, December 2005.

[8] S. D. Kamvar, M. T. Schlosser, and H. Garcia-Molina. The eigentrust algorithm for reputation management in $\mathrm{P} 2 \mathrm{P}$ networks. In Proceedings of the 12th International WWW Conference, Budapest, Hungary, May 2003.

[9] D. H. Knight and N. L. Chervany. The meaning of trust. Technical Report WP9604, Universoty of Minnesota, Management Information Systems Research Center, 1996.

[10] K.-J. Lin, H. Lu, T. Yu, and C. en Tai. A reputation and trust management broker framework for web applications. In Proceedings of The 2005 IEEE International Conference on e-Technology, e-Commerce and e-Service (EEE'05), pages 262-269, March 2005.

[11] S. Marsh. Formalising Trust as a Computational Concept. University of Stirling, 1994.

[12] S. Marti and H. Garcia-Molina. Limited reputation sharing in P2P systems. In Proceedings of ACM EC'04, pages 91101, New York, USA, May 2004.

[13] I. Ray and S. Chakraborty. A vector model of trust for developing trustworthy systems. In Proceedings of ESORICS 2004, volume LNCS 3193, Springer-Verlag, pages 260-275, 2004.

[14] J. Sabater and C. Sierra. REGRET: A reputation model for gregarious societies. In Proceedings of the First International Joint Conference on Autonomous Agents in MultiAgent Systems (AAMAS-02), pages 475-482, 2002.

[15] R. S. Sandhu, E. J. Coyne, H. L. Feinstein, and E. Youma. Role-based access control models. IEEE Computer, 29:3847, Feb. 1996.

[16] L.-H. Vu, M. Hauswirth, and K. Aberer. Qos-based service selection and ranking with trust and reputation management. In Proceedings of 13th International Conference on Cooperative Information Systems (CoopIS 2005), Oct 31-Nov 4 2005.

[17] Y. Wang and V. Varadharajan. Interaction trust evaluation in decentralized environments. In K. Bauknecht, M. Bichler, and B. Pröll, editors, Proceedings of 5th International Conference on Electronic Commerce and Web Technologies (EC-Web04), volume LNCS 3182, Springer-Verlag, pages 144-153, Zaragoza, Spain, August-September 2004.

[18] Y. Wang and V. Varadharajan. Trust ${ }^{2}$ : Developing trust in peer-to-peer environments. In Proceedings of 2005 IEEE International Conference on Services Computing (SCC 2005), pages 24-31, Orlando, Florida, USA, July 2005.

[19] S. Wasserman and K. Faust. Social Network Analysis. Cambridge University Press, 1994.

[20] B. Xiao, E. Aimeur, and J. M. Fernandez. Pcfinder: An intelligent product recommendation agent for e-commerce. In Proceedings of 2003 IEEE International Conference on ECommerce Technology (CEC'03), pages 181-188, Newport Beach, CA, USA, 24-27 June 2003.

[21] L. Xiong and L. Liu. PeerTrust: Supporting reputationbased trust for peer-to-peer electronic communities. IEEE Trans. on Knowlege and Data Engineering, 16(7):843-857, 2004. 